This is an Open Access article distributed under the terms of the Creative Commons Attribution-Noncommercial License, which permits unrestricted use, distribution, and reproduction in any noncommercial medium, provided the original work is properly cited.

\title{
MILLIMETRE AND FIR BROADBAND QUASI OPTICAL DEVICES
}

\section{Haynes, B. Maffei, S. J. Melhuish, L. Piccirillo, G. Pisano, and D. Shakeshaft}

Jodrell Bank Centre for Astrophysics, Alan Turing Building, School of Physics and Astronomy, The University of Manchester, Oxford Road, Manchester M13 9PL, UK

\section{ABSTRACT}

We present a set of techniques and materials we are currently developing which enable very broadband and highly effective optical devices in the spectral region from $20 \mathrm{GHz}$ to $20 \mathrm{THz}$. Many of these devices have already been employed in terrestrial, airborne and space based telescope systems.

Key words: Rutile, Antireflection coating, Broadband, Far Infrared, Millimetre Wave, Sub Millimetre Wave, Wave Plate, Cryostat Window, Filter, Lens, Quartz, Sapphire, Silicon, Thermal Control

\section{GRIDS FOR FILTERS AND POLARIZERS}

We have developed Ulrich type, metal grid embedded in polymer dielectric, filters for the range $20 \mathrm{GHz}$ to $20 \mathrm{THz}$ (see Figures 1 and 2). Other devices using the same technology such as wave plates and polarizers are being developed. Methods are being developed to reduce the bandwidth limitations of lithographic polarizers. This involves improved fine detail lithography for higher frequencies while thicker metal reduces low frequency skin depth problems. The fabrication techniques of all these devices are consistent with large diameters as required for the next generation of large aperture detector array instruments.

\section{ANTIREFLECTION COATINGS}

\subsection{CoAtings FOR High-INDEX MATERIALS}

Antireflection coating naturally divides into two categories, high substrate index coatings for crystalline materials such as semiconductors or ionic solids and low substrate index coatings for polymers. For the high index coatings we have developed a material with a tunable refractive index $(1.5-2.7)$ which can be used to realize theoretically optimal multilayer coatings (see Figure 3 ). This consists of nanoparticle rutile grains dispersed in polypropylene in the same manner as a Yoshinaga type filter. Multilayers of this and other materials enable very broad and efficient antireflection coating. This has enabled the use of synthetic sapphire for Pancharatnam type waveplates and larger waveplates for which suitable crystalline quartz is not affordable or even available (Figure 4). Other devices which benefit from this technology include cryostat windows and lenses.

\subsection{CoATINGS FOR LOW-INDEX MATERIALS}

Broadband antireflection coatings are currently being developed for low refractive index polymer substrates. The range of suitable materials is more limited here as even lower indices than the substrate are required. Some usable polymers are available and very low indices are achieved using microporous polymers (see Figure 5) but we do not have the same freedom as with a tunable index material. These techniques are usable with the above mentioned Ulrich type filters and other metal grid embedded devices. Other applications include polymeric devices such as cryostat windows and lenses (Figure 6).

\section{VERY LARGE CRYOSTAT WINDOWS}

Techniques for developing very large diameter cryostat windows are being developed in order to cope with the trends towards using larger array detectors and putting more of the optics in the cryostat. Physical reliability of these devices, which may have to take loads of several tonnes, is paramount as a lot of expensive equipment is usually at stake. Long term quantitative pressure testing is required to assess ageing characteristics rather than just a quick over pressure test. An unfortunate characteristic of large aperture windows is that they admit large thermal loads to the cryostat interior. Passive cascading techniques are being developed to reduce this thermal loading without significant degradation of the required signal and with the added benefit of reducing condensation on the windows outer surface. 


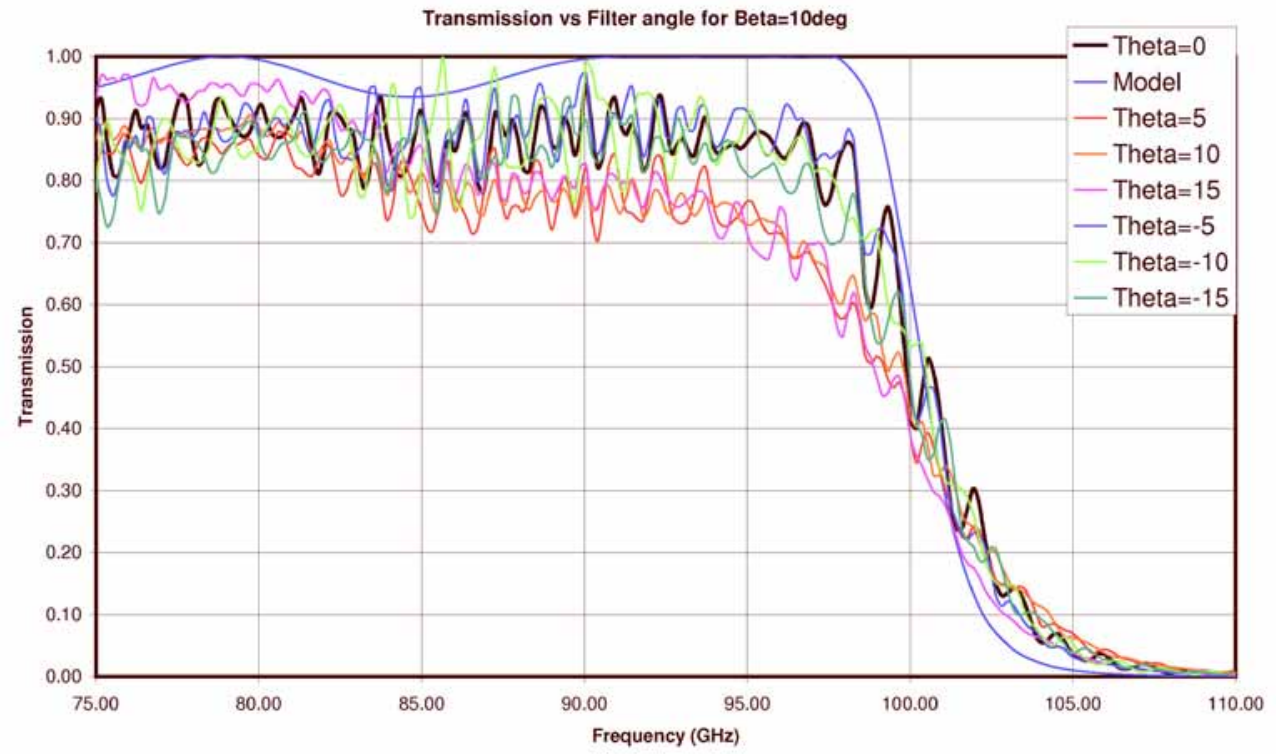

Figure 1. Modeled and measured data for $100 \mathrm{GHz}$ low pass edge filter.

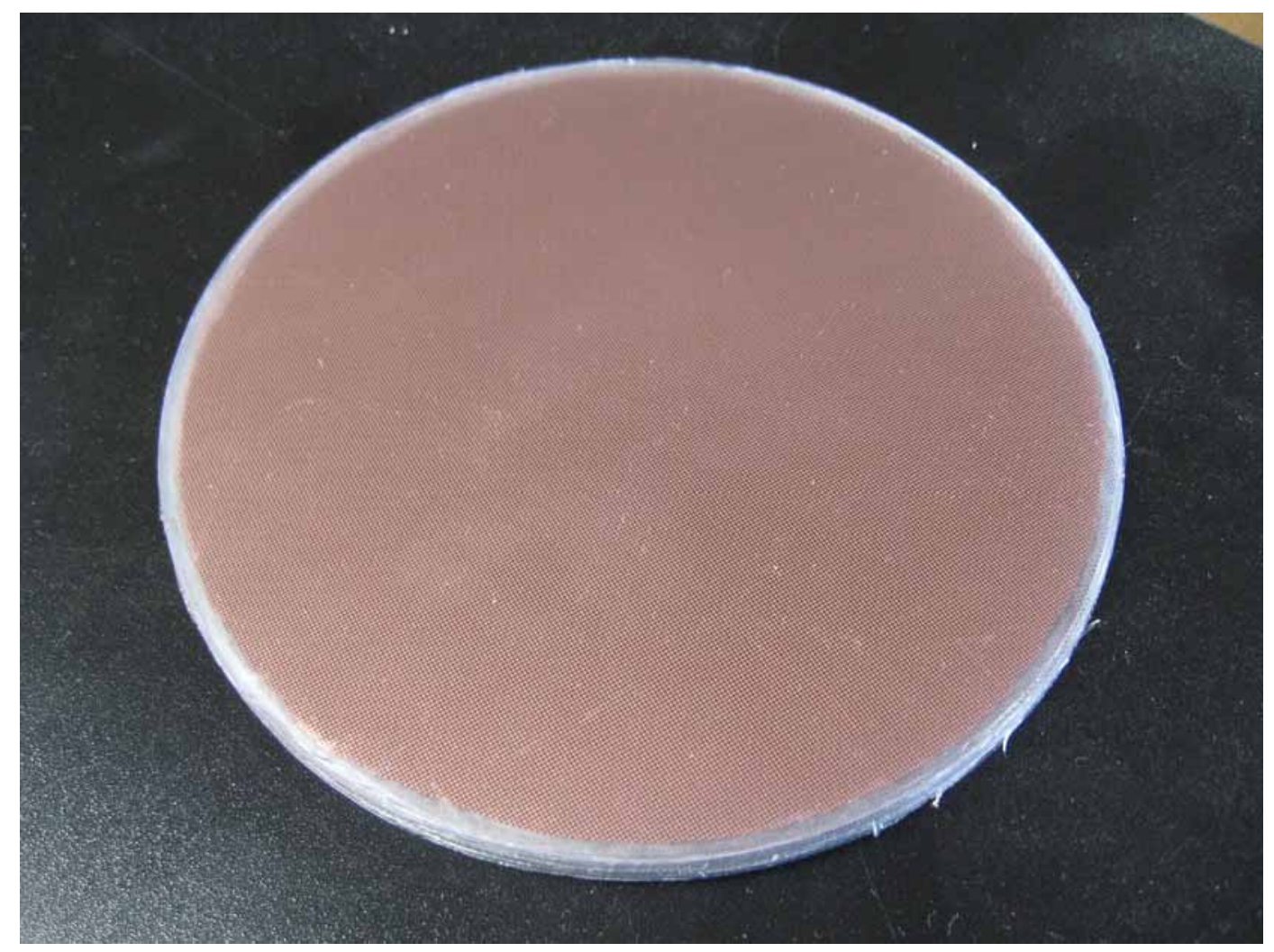

Figure 2. Ulrich type grid filter embedded in polypropylene. 
Z-cut quartz (thickness $5.73 \mathrm{~mm}$ )

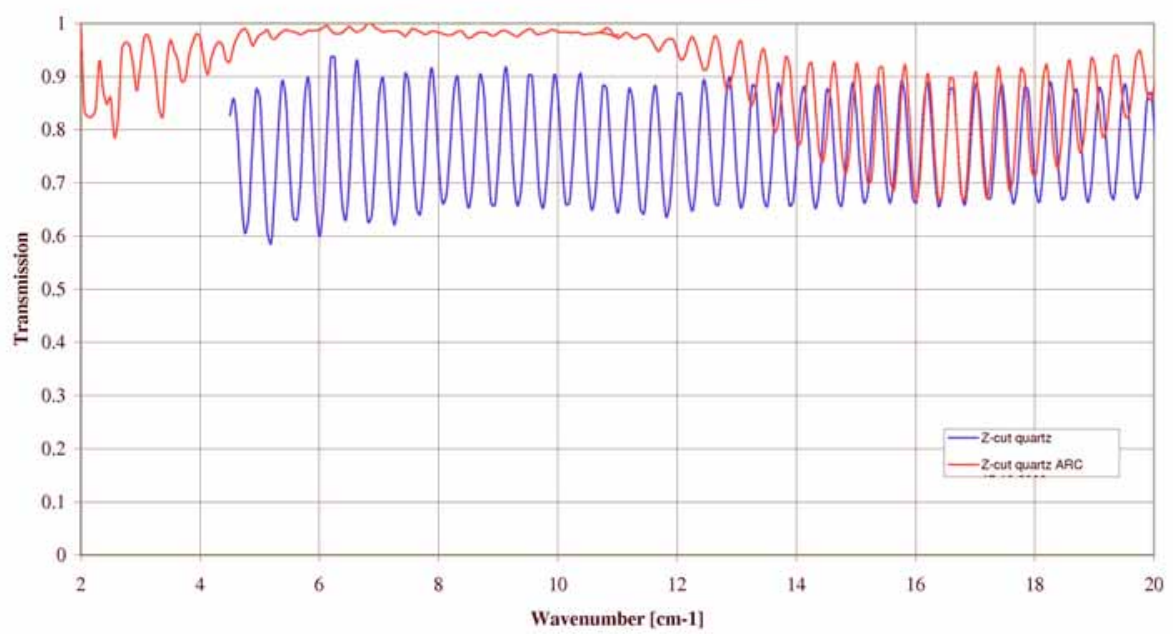

Figure 3. Crystalline quartz window before and after antireflection coating (measured at Cardiff university).

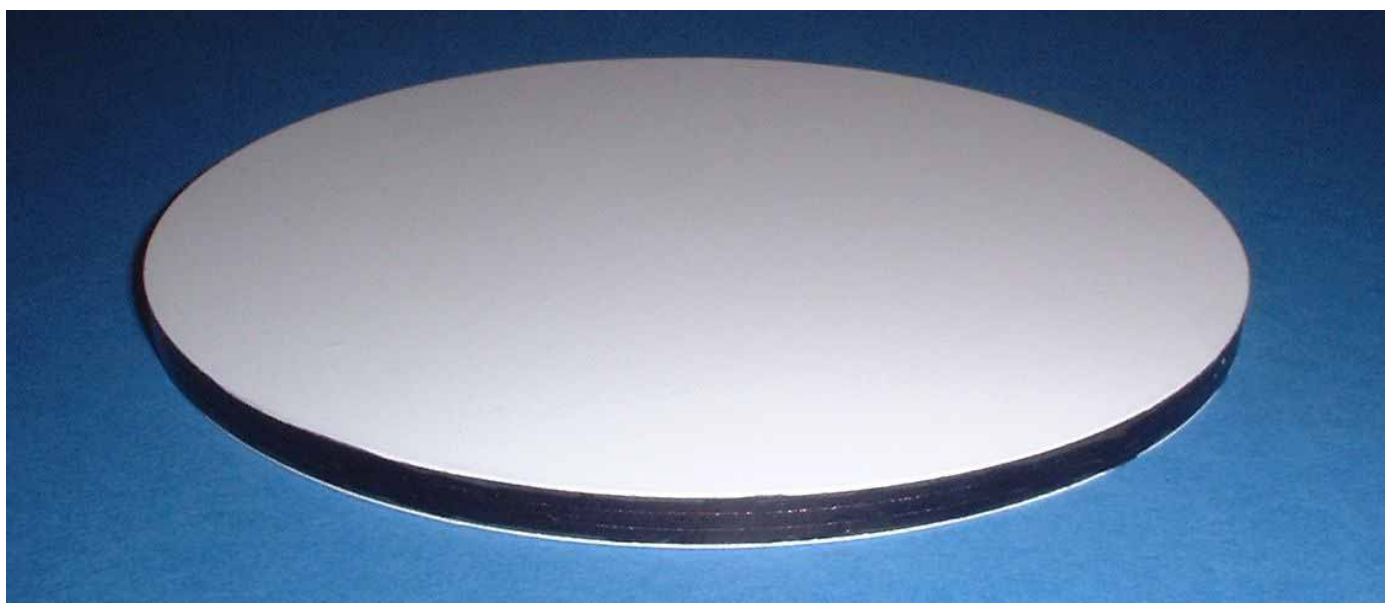

Figure 4. Sapphire Pancharatnam waveplate with three layer coatings (made at Cardiff university) 
One octave demonstration design on polyethylene

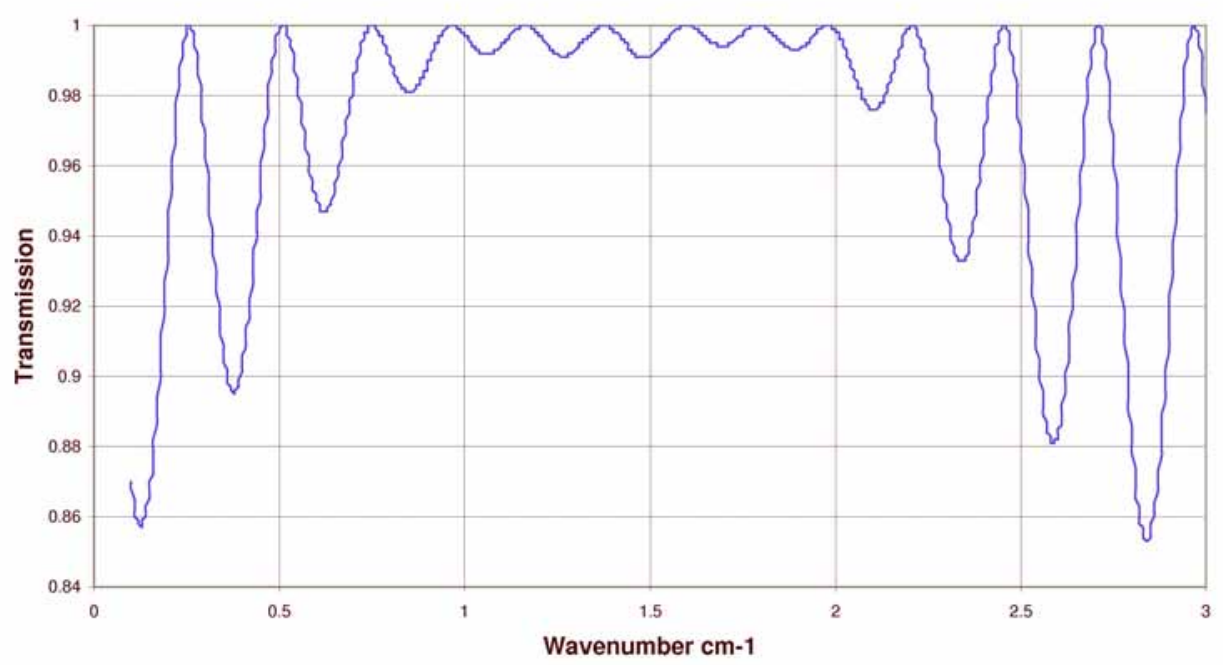

Figure 5. Modeled two layer broadband coating for polyethylene substrate.

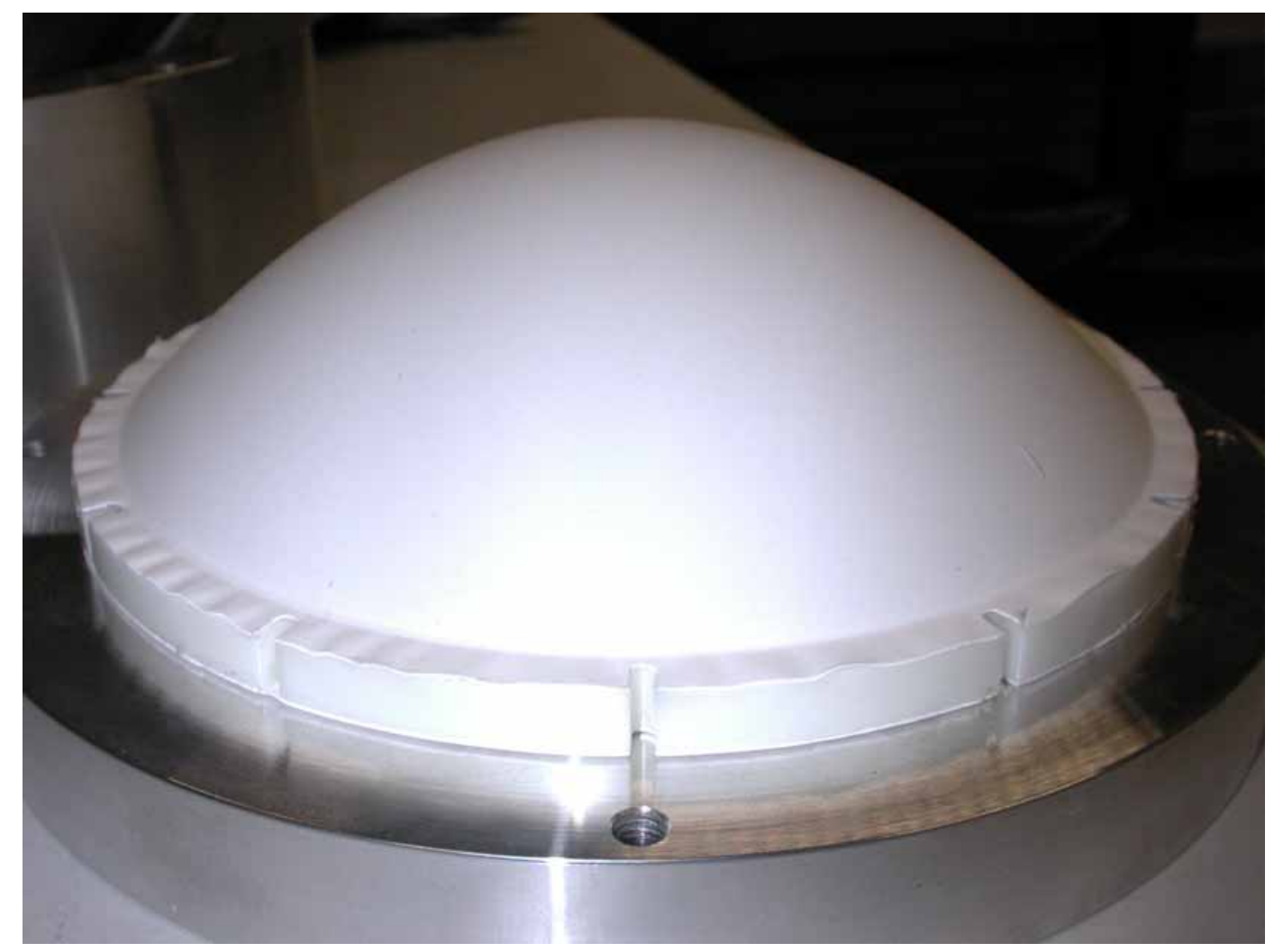

Figure 6. Coated polyethylene lens used in QUAD (made at Cardiff university). 